\title{
Survey on Optimization of The Energy Consumption and Load Balancing in Wireless Sensor Network
}

\author{
Khairul Nadilah Bt Z. A. ${ }^{1}$ and Sazlinah Hasan ${ }^{2}$ \\ ${ }^{1,2}$ Faculty of Computer Science and Information Technology, \\ Universiti Putra Malaysia (UPM), Malaysia \\ 1dilanaddy@gmail.com, 22sazlinah@upm.edu.my
}

\begin{abstract}
In era Internet of Thing (IoT), Wireless Sensor Network (WSN) is the most important device involved in communication networks between two or more nodes [1]. The growth of communication in the network may cause the performance of delivering packets to become slower or it could be loss packets during transmission time. There are many factors need to be considered in order to decrease the packet loss such as energy consumption, memory space, load balancing, network lifetime, etc. The main focus in this paper is to identify the most problematic issues that happen in the Wireless Sensor Network which is focus on balance heavy traffic load and the energy consumption on the network system. Some comparison will be recognized based on several reviewed research paper and will show the strength and limitation on Wireless Sensor Network communication.
\end{abstract}

Key words - Energy Consumption, Load Balancing, Node, Network Lifetime, Packet Loss, Wireless Sensor Networks.

\section{INTRODUCTION}

Technologies Internet of Thing nowadays towards into the Industry 4.0, also known as a smart industry or Fourth Industrial Revolution (4IR). It is involving with some group of sensors, communication hardware, embedded software, Cloud Computing Service and the manufacturing that related with electronic system to share, monitoring, collection, recorded, communication, also as a transportation data [2].

Wireless Sensor Network is one of the innovative technologies of the Internet of Things that make all the equipment or device can communicate with each other [3]. It is getting more attention from various media site, either it from companies, entrepreneurs, government, military and also civil society. Many devices have been created to meet demand target for advanced technology goes smoothly and well organized.

In Wireless Sensor Network, data generated in a network with consist lot of sensor nodes, all these nodes monitored by the central entity as a special node call as Sink Node or Base Station (BS) shown in Figure 1, where all nodes interact with each other used a frequency signal of radio. BS collects all the data and process it and send the available data to the consumer via the Internet [5]. Meanwhile, based on energy consumption concept, energy will increase due to the incensement of communication distance. The data that close to the sink should be pass to other nodes. This will make energy drain faster and lead to the creation of whole energy around the sink. As an outcome, the whole network indicates early death caused by the creation of an energy hole. This sentence is supported by several authors [6]-[9].

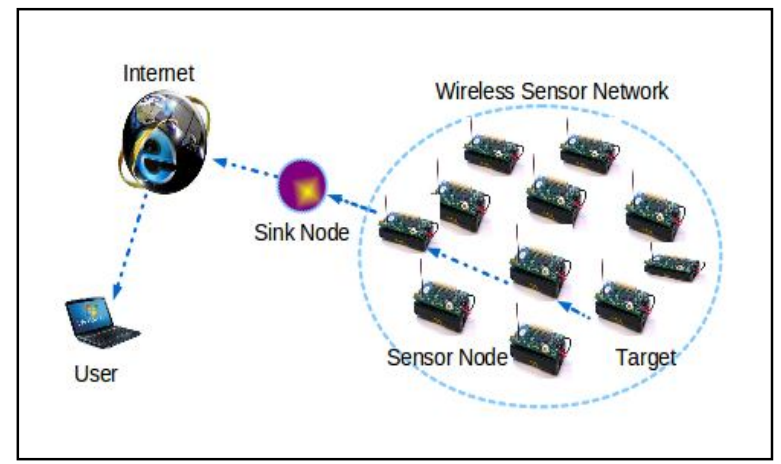

Figure 1: The Wireless Sensor Network [4]

The biggest technology growth in the scope of Wireless Sensor Networks will build a group or thousands of connections between nodes in the communication system. These issues will lead to the creation of some factor towards on failure of WSN. There are several factors of weakness in WSN such as high energy consumption, congestion traffic, unbalance load, slow data processing, compressing techniques, cross-layer design, communication arrangement, thousands of nodes creation, etc [10]. All of these may be caused by heat, electromagnetic interference, equipment problem, multi-path effect, human error, dust, noise, and fading, which make WSNs limited to give the consumer the best performance service [11].

The following issues in the proposed survey paper follow as: In section 2, Related work of Wireless Sensor Network will be presented. Taxonomy of existing work on Wireless Sensor Network will be reviewed in section 3., strength and 
limitation in section 4 and Section 5 will present the conclusion and future research.

\section{RELATED WORK}

This survey paper will identify most of the strength and weakness of the Wireless Sensor Network. But before that, all the related to WSN need to study first.

In a Wireless Sensor Network, the ability of transmission and receiver relies on node energy and make energy usage have a limited lifetime. The drain of energy may cause a loss of network data in the transmission line [7]. Some existing approaches said that most sensor nodes used power by a limited energy source such as a battery that means it not easy to continue to sustain the lifetime expectancy.

There are several approaches technique have been done by some author to implement the weaknesses in the communication network. As in [9], their proposed energyaware balanced energy consuming and hole alleviating algorithm (EA_BECHA) to minimize the data drop ratio to increase the throughput. The difference with [6] and [12], where both adopt the same technique that is the sleep-wake schedule algorithm to save and minimized the maximum energy on sensor nodes. However, there are also have another way to solve the problem on high energy consumption such as part energy control technique (PECT) which setting the node energy based on path energy consumption to propose by [2], energy balance routing protocol (EBRP) propose by [13] to protect the low energy node through a compact energy area for sent nodes to the sink.

As mention in energy consumption section above, unbalance load also can cause a problem of the premature deaths in earlier than other nodes. Unbalance traffic load may happen when used more one sensor nodes in the transmission range. Which mean all the nodes will focus at the same point at the same time. This will cause of creation hole around the node sink or base station where the hole occurred because of extreme heat concentrated there.

Previous research has shown by change the range of intermediate network nodes at different distances to the sink for bypass the high load region will make energy usage can be distributed and can continue to extend the lifetime [14]. The author has proposed a minimal-energy part, balanced communication on nodes, and ultimately lead to failure network on premature nodes. Same as [12], the author used to create balance dynamic change for traffic load to find a route with an available channel to sink nodes. Although some researchers approach another solution which is presented a Dynamic Hyper Round Policy (DHRP) schedules which cluster static task to split a time to fix length rounds at the beginning of the performance. The schedule is to obtain a load balancing of network lifetime [15]. Almost similar to the DHRP approach, in [1] use cluster algorithms to cluster the size of routing to increase network lifetime. In [16], presents a Low power and Lossy network (LLN) routing to control the heavy dynamic load which designs a low throughput network.

All of this case study can be concluded that both energy consumption and balance traffic load the most significant to each other in order to implement a Wireless Sensor Network. Both have the same issues and the same structure that need to be modify become more stable.

\section{TAXONOMY OF COMPARATIVE ISSUES IN WSN}

Based on the previous Section 2, all the review paper can be classification into several criteria with differences entity. Table. 1 shows that frequent issues occur in Wireless Sensor Network. Instead, there are many ways that can be addressed to fix related issues. There is comparative work, strength, limitation of some literature reviews as shown in Table 1.

Table 1: Taxonomy of Existing Work

\begin{tabular}{|c|c|c|c|c|c|c|c|}
\hline \multirow{2}{*}{$\begin{array}{l}\text { Author, } \\
\text { Year }\end{array}$} & \multirow[t]{2}{*}{ Ref } & \multirow{2}{*}{ Contribution } & \multicolumn{5}{|c|}{ Comparison Criteria } \\
\hline & & & $\begin{array}{c}\text { Control } \\
\text { Issues }\end{array}$ & $\begin{array}{c}\text { Algorithm } \\
\text { / Classifier } \\
\text { used }\end{array}$ & $\begin{array}{c}\text { Method/ } \\
\text { Tools }\end{array}$ & Strength & Limitation \\
\hline $\begin{array}{l}\text { J. Zhang, } \\
\text { X. Feng, } \\
\text { and Z. } \\
\text { Lui, } 2018\end{array}$ & {$[1]$} & $\begin{array}{l}\text { - Analyzing load distribution } \\
\text { model and energy } \\
\text { consumption. } \\
\text { - The cluster size of nodes to } \\
\text { balance energy } \\
\text { consumption. } \\
\text { - Cluster head by selected } \\
\text { energy and distance in each } \\
\text { cluster. }\end{array}$ & $\begin{array}{l}- \text { Load } \\
\text { Balance } \\
\cdot \text { Energy } \\
\text { Efficient } \\
- \text { Cluster } \\
\text { Nodes }\end{array}$ & $\begin{array}{l}\text { - GCA } \\
\cdot \text { ACT } \\
\cdot \text { ER-HEED } \\
\cdot \text { RUHEED }\end{array}$ & Simulation & $\begin{array}{l}\text { - Minimize } \\
\text { energy } \\
\text { consumption. } \\
\text { - Balance energy } \\
\text { of cluster head. } \\
\text { - Minimize } \\
\text { overhead } \\
\text { communication. }\end{array}$ & $\begin{array}{l}\text { High energy } \\
\text { consumption. } \\
\text { - Unbalance } \\
\text { energy cluster. } \\
\text { - Unbalance load } \\
\text { distribution. }\end{array}$ \\
\hline $\begin{array}{l}\text { F. } \\
\text { Tubiello } \\
\text { et al., } \\
2018\end{array}$ & {$[2]$} & $\begin{array}{l}\text { - Decreases the WSN } \\
\text { efficiency to increase } \\
\text { energy consumption. } \\
\text { - Reduce energy } \\
\text { consumption in } \\
\text { transmission data. } \\
\text { - Increase the lifetime }\end{array}$ & $\begin{array}{l}- \text { Energy } \\
\text { Efficient } \\
\text { - Lifetime } \\
\text { Network }\end{array}$ & $\begin{array}{l}\cdot \text { - DPR } \\
\text { - EEOR } \\
\text { - PECT } \\
\text { - QPth } \\
\text { - RSSI }\end{array}$ & Simulator & $\begin{array}{l}\text { - Reduce energy } \\
\text { consumption. } \\
\text { - Increase lifetime } \\
\text { nodes. } \\
\text { - Increase packet } \\
\text { retransmission. }\end{array}$ & $\begin{array}{l}\text { - High Packet } \\
\text { loss. } \\
\text { - High energy } \\
\text { consumption. } \\
\text { - Limited } \\
\text { memory } \\
\text { capacity. }\end{array}$ \\
\hline
\end{tabular}




\begin{tabular}{|c|c|c|c|c|c|c|c|}
\hline \multirow{2}{*}{$\begin{array}{l}\text { Author, } \\
\text { Year }\end{array}$} & \multirow[t]{2}{*}{ Ref } & \multirow[t]{2}{*}{ Contribution } & \multicolumn{5}{|c|}{ Comparison Criteria } \\
\hline & & & $\begin{array}{l}\text { Control } \\
\text { Issues }\end{array}$ & $\begin{array}{c}\text { Algorithm } \\
\text { / Classifier } \\
\text { used }\end{array}$ & $\begin{array}{l}\text { Method/ } \\
\text { Tools }\end{array}$ & Strength & Limitation \\
\hline & & network. & & & & & \\
\hline $\begin{array}{l}\text { S. Kaur } \\
\text { and R. } \\
\text { Goel, } \\
2016\end{array}$ & {$[5]$} & $\begin{array}{l}\text { - Clustering protocol energy- } \\
\text { efficient communication } \\
\text { protocol where energy } \\
\text { conservation needs. } \\
\text { - Selection node for cluster } \\
\text { head, } \\
\text { - Responsible to collect } \\
\text { sensed data from own } \\
\text { cluster. } \\
\text { - Aggregate the data to the } \\
\text { base station or sink. } \\
\end{array}$ & $\begin{array}{l}- \text { Energy } \\
\text { Efficient } \\
\text { - Cluster } \\
\text { Nodes }\end{array}$ & $\begin{array}{l}-\mathrm{LEACH} \\
\cdot \mathrm{EEHC} \\
\cdot \mathrm{EECP} \\
\cdot \mathrm{DEEC} \\
\cdot \mathrm{G}-\mathrm{DEEC} \\
\cdot \mathrm{M}-\mathrm{GEAR} \\
\cdot \mathrm{GBC}\end{array}$ & $\begin{array}{c}\text { Review } \\
\text { Paper }\end{array}$ & $\begin{array}{l}\text { - Cluster } \\
\text { algorithm to } \\
\text { perform energy } \\
\text { stability. } \\
\text { - Better utilization } \\
\text { and stability } \\
\text { lifetime. }\end{array}$ & $\begin{array}{l}\text { Energy } \\
\text { unstable on a } \\
\text { cluster node. } \\
\text { - Short lifetime. }\end{array}$ \\
\hline $\begin{array}{l}\text { C. Zhan, } \\
\text { Y. Zeng, } \\
\text { and R. } \\
\text { Zhang, } \\
2018\end{array}$ & [6] & $\begin{array}{l}\text { - Sensor Nodes wake-up } \\
\text { schedule and UAV's } \\
\text { trajectory to achieve } \\
\text { reliable and energy- } \\
\text { efficient. } \\
\text { - Minimize energy } \\
\text { consumption for all SN's } \\
\text { - Design mixed-integer non- } \\
\text { convex. } \\
\text { - Compare benchmark } \\
\text { scheme with static data. }\end{array}$ & $\begin{array}{l}- \text { Energy } \\
\text { Efficient } \\
- \text { Load } \\
\text { Balance. }\end{array}$ & $\begin{array}{l}\cdot \text { UAV- } \\
\text { Enabled }\end{array}$ & $\begin{array}{l}\text { CVX - a } \\
\text { Software } \\
\text { tool }\end{array}$ & $\begin{array}{l}\text { - Minimized the } \\
\text { maximum } \\
\text { energy. } \\
\text { consumption } \\
\text { - Wake-up } \\
\text { schedule stable } \\
\text { and efficient. }\end{array}$ & $\begin{array}{l}\text { - Strike a } \\
\text { balancing on } \\
\text { the direct link } \\
\text { and } \\
\text { interference } \\
\text { link. } \\
\text { - High Energy } \\
\text { Consumption. }\end{array}$ \\
\hline $\begin{array}{l}\text { I. Khan, } \\
\text { and D. } \\
\text { Singh, } \\
2018\end{array}$ & [7] & $\begin{array}{l}\text { - Analysis of selection } \\
\text { probability and energy } \\
\text { balance of node path. } \\
\text { - Determine the energy node: } \\
\text { - Selected the maximum } \\
\text { distance and energy. } \\
\text { - Consider the shorted path, } \\
\text { direction, energy } \\
\text { adjustment for the selected } \\
\text { node. } \\
\text { - Filter algorithm: } \\
\text { - Eliminate the path node } \\
\text { by used dynamic } \\
\text { selected and balancing } \\
\text { the energy. } \\
\text { - To prove network lifetime, } \\
\text { stability and accuracy need } \\
\text { to perform in data analysis. }\end{array}$ & $\begin{array}{l}- \text { Energy } \\
\text { Efficient } \\
\text { - Distance } \\
\text { Nodes }\end{array}$ & - EBDA & MATLAB & $\begin{array}{l}\text { - Optimizes } \\
\text { energy network } \\
\text { also probability } \\
\text { nodes. } \\
\text { - Improve lifetime } \\
\text { node. }\end{array}$ & $\begin{array}{l}\text { - Low accuracies } \\
\text { and data rate } \\
\text { during the pre- } \\
\text { operation. } \\
\text { - Unbalance } \\
\text { Load Energy. } \\
\text { - Fast drain } \\
\text { energy on node } \\
\text { transmission. }\end{array}$ \\
\hline $\begin{array}{l}\text { J. Ren et } \\
\text { al., } 2016\end{array}$ & [8] & $\begin{array}{l}\text { - Estimation of energy } \\
\text { consumption, load } \\
\text { balancing, and sensor nodes } \\
\text { lifetime. } \\
\text { - A lifetime of dead nodes } \\
\text { - Estimate error in less than } \\
\text { 5\% rate. } \\
\text { - Studies on the energy hole } \\
\text { of temporal and spatial also } \\
\text { provided theoretical } \\
\text { mitigate energy hole. }\end{array}$ & $\begin{array}{l}- \text { Load } \\
\text { Balance. } \\
\text { - Energy } \\
\text { Efficient } \\
\text { - Life } \\
\text { Node. }\end{array}$ & $\begin{array}{l}\text { - FNDT } \\
\text { - ANDT }\end{array}$ & OMNET++ & $\begin{array}{l}- \text { Reduce error } \\
\text { rate energy hole } \\
\text { to }<5 \% \\
\text { - Improve } \\
\text { network lifetime }\end{array}$ & $\begin{array}{l}\text { - Short energy } \\
\text { lifetime on the } \\
\text { node. } \\
\text { - Creation energy } \\
\text { hole. } \\
\text { - Unbalance } \\
\text { energy } \\
\text { consumption. }\end{array}$ \\
\hline
\end{tabular}


Khairul Nadilah Bt Z. A et al., International Journal of Advanced Trends in Computer Science and Engineering, 8(1.4), 2019, 229- 235

\begin{tabular}{|c|c|c|c|c|c|c|c|}
\hline \multirow{2}{*}{$\begin{array}{l}\text { Author, } \\
\text { Year }\end{array}$} & \multirow[t]{2}{*}{ Ref } & \multirow[t]{2}{*}{ Contribution } & \multicolumn{5}{|c|}{ Comparison Criteria } \\
\hline & & & $\begin{array}{l}\text { Control } \\
\text { Issues }\end{array}$ & $\begin{array}{c}\text { Algorithm } \\
\text { / Classifier } \\
\text { used }\end{array}$ & $\begin{array}{l}\text { Method/ } \\
\text { Tools }\end{array}$ & Strength & Limitation \\
\hline $\begin{array}{l}\text { N. Jan et } \\
\text { al., } 2017\end{array}$ & [9] & $\begin{array}{l}\text { - Balancing the load of the } \\
\text { network. } \\
\text { - Enhance throughput } \\
\text { - The packet drop ratio is } \\
\text { minimized. } \\
\text { - To efficiently the } \\
\text { utilizing of the energy } \\
\text { the lifetime has been } \\
\text { prolog. }\end{array}$ & $\begin{array}{l}- \text { Load } \\
\text { Balance } \\
\text { - Energy } \\
\text { Efficient }\end{array}$ & $\begin{array}{l}\cdot-\mathrm{WSNEHA} \\
\cdot \text { EA- } \\
\text { BECHA }\end{array}$ & Simulation & $\begin{array}{l}\text { - Minimized load } \\
\text { and energy hole } \\
\text { level. } \\
\text { - Maximize the } \\
\text { packet delivery. } \\
\text { - Energy } \\
\text { constraint in the } \\
\text { transmission } \\
\text { process. }\end{array}$ & $\begin{array}{l}\cdot \text { High } \\
\text { bandwidth. } \\
\cdot \text { High residual } \\
\text { energy. }\end{array}$ \\
\hline $\begin{array}{l}\text { M. } \\
\text { Faheem, } \\
\text { and V.C. } \\
\text { Gungor, } \\
2017\end{array}$ & [11] & $\begin{array}{l}\text { - Dynamic cluster algorithm: } \\
\text { - Balance traffic load and } \\
\text { energy consumption. } \\
\text { - BMO clustering routing: } \\
\text { - Balance energy on } \\
\text { cluster head. } \\
\text { - Propose protocol to reduce } \\
\text { end to end delay. } \\
\text { - Improve throughput, } \\
\text { memory, packet delivery, } \\
\text { and remaining energy. }\end{array}$ & $\begin{array}{l}- \text { Load } \\
\text { Balance. } \\
\text { - Energy } \\
\text { Efficient } \\
\text { - Cluster } \\
\text { Routing. } \\
\text { - Routing } \\
\text { Protocol }\end{array}$ & $\begin{array}{l}\cdot \mathrm{BMO} \\
\cdot \mathrm{EQRP}\end{array}$ & EstiNet9.0 & $\begin{array}{l}\text { - Balance data } \\
\text { traffic. } \\
\text { - Maximize } \\
\text { energy efficient. } \\
\text { - Control packet } \\
\text { loss. } \\
\text { - Maximum } \\
\text { lifetime energy. }\end{array}$ & $\begin{array}{l}\text { - Imbalance } \\
\text { energy } \\
\text { consumption. } \\
\text { - Nonuniform } \\
\text { node } \\
\text { distribution. } \\
\text { - Data path loop. } \\
\text { - Packet loss. } \\
\text { - High delay. }\end{array}$ \\
\hline $\begin{array}{l}\text { X. Li et } \\
\text { al., } 2017\end{array}$ & [12] & $\begin{array}{l}\text { - Change time an identified } \\
\text { using SVM to balance the } \\
\text { traffic. } \\
\text { - Maximum traffic load: } \\
\text { - Used DGA to obtain a } \\
\text { good traffic balance } \\
\text { level. } \\
\text { - Deploy the cluster head } \\
\text { node. } \\
\text { - Sleep-wake schedule to } \\
\text { control energy } \\
\text { consumption. }\end{array}$ & $\begin{array}{l}- \text { Load } \\
\text { Balance } \\
\text { - Energy } \\
\text { Efficient } \\
\text { - Cluster } \\
\text { Node }\end{array}$ & $\begin{array}{l}\cdot \text { SDSS } \\
\cdot \text { CHN } \\
\cdot \text { SHTGT }\end{array}$ & Simulation & $\begin{array}{l}\text { - Control energy } \\
\text { consumption } \\
\text { used the sleep- } \\
\text { wake technique. } \\
\text { - Minimized } \\
\text { heavy load. }\end{array}$ & $\begin{array}{l}\cdot \text { High energy } \\
\text { consumption. } \\
\text { - Unbalance } \\
\text { traffic load. }\end{array}$ \\
\hline $\begin{array}{l}\text { F. Ren et } \\
\text { al., } 2011\end{array}$ & [13] & $\begin{array}{l}\text { - Energy balance data } \\
\text { gathering routing algorithm. } \\
\text { - Construct an independent } \\
\text { virtual field. } \\
\text { - Protect the low energy } \\
\text { nodes for residual energy. }\end{array}$ & $\begin{array}{l}- \text { Energy } \\
\text { Efficient } \\
\text { - Load } \\
\text { Balance }\end{array}$ & $\begin{array}{l}-\mathrm{EBRP} \\
-\mathrm{RSSI} \\
-\mathrm{CLP}\end{array}$ & Tiny-OS & $\begin{array}{l}\text { - Stable energy } \\
\text { consumption. } \\
\text { - Maximized } \\
\text { network } \\
\text { lifetime. } \\
\text { - Minimized } \\
\text { heavy load. }\end{array}$ & $\begin{array}{l}\text { - Low energy } \\
\text { lifetime. } \\
\text { - High energy } \\
\text { consumption. } \\
\text { - Unbalance } \\
\text { energy node. } \\
\text { - Lack of } \\
\text { dynamic time- } \\
\text { varying } \\
\text { potential field. }\end{array}$ \\
\hline $\begin{array}{l}\text { K. Lui, } \\
\text { and C. } \\
\text { Wang, } \\
2017\end{array}$ & {$[14]$} & $\begin{array}{l}\text { - Minimize energy } \\
\text { consumption path. } \\
\text { - Balance load of nodes. } \\
\text { - Improve the network } \\
\text { lifetime. }\end{array}$ & $\begin{array}{l}- \text { Load } \\
\text { Balance } \\
\text { - Energy } \\
\text { Efficient }\end{array}$ & $\begin{array}{l}- \text { ORECA } \\
\text { - ROL } \\
\text { - MORA } \\
\text { - EARP }\end{array}$ & NS-2 & $\begin{array}{l}\text { - Minimize } \\
\text { energy } \\
\text { consumption } \\
\text { - Balance } \\
\text { communication } \\
\text { of nodes }\end{array}$ & $\begin{array}{l}\text { - High energy } \\
\text { consumption. } \\
\text { - Limited } \\
\text { lifetime battery. } \\
\text { - High delay } \\
\text { process. } \\
\text { - Overhead of } \\
\text { the route. }\end{array}$ \\
\hline $\begin{array}{l}\text { P. } \\
\text { Neamatoll }\end{array}$ & [15] & $\begin{array}{l}\text { - Cluster task - schedules } \\
\text { load balancing. }\end{array}$ & $\begin{array}{l}- \text { Load } \\
\text { Balance. }\end{array}$ & $\begin{array}{l}- \text { DHRP } \\
-\mathrm{RBP} \\
\end{array}$ & MATLAB & $\begin{array}{l}\text { - Maximized the } \\
\text { network }\end{array}$ & $\begin{array}{l}- \text { Overhead due } \\
\text { to clustering. }\end{array}$ \\
\hline
\end{tabular}


Khairul Nadilah Bt Z. A et al., International Journal of Advanced Trends in Computer Science and Engineering, 8(1.4), 2019, 229- 235

\begin{tabular}{|c|c|c|c|c|c|c|c|}
\hline \multirow{2}{*}{$\begin{array}{l}\text { Author, } \\
\text { Year }\end{array}$} & \multirow[t]{2}{*}{ Ref } & \multirow[t]{2}{*}{ Contribution } & \multicolumn{5}{|c|}{ Comparison Criteria } \\
\hline & & & $\begin{array}{l}\text { Control } \\
\text { Issues }\end{array}$ & $\begin{array}{c}\text { Algorithm } \\
\text { / Classifier } \\
\text { used }\end{array}$ & $\begin{array}{l}\text { Method/ } \\
\text { Tools }\end{array}$ & Strength & Limitation \\
\hline $\begin{array}{l}\text { ahi et al., } \\
2018\end{array}$ & & $\begin{array}{l}\text { - Eliminates the unnecessary } \\
\text { by re-cluster. } \\
\text { - Control throughput network } \\
\text { lifetime. } \\
\text { - Enhance the cluster task by } \\
\text { online and offline } \\
\text { algorithms. }\end{array}$ & $\begin{array}{l}- \text { Energy } \\
\text { Efficient } \\
\cdot \text { Life } \\
\text { Node. }\end{array}$ & & & $\begin{array}{l}\text { lifetime. } \\
\text { - Balance energy } \\
\text { efficient. } \\
\text { - Reduce energy } \\
\text { consumption. }\end{array}$ & $\begin{array}{l}\text { - Wasted energy } \\
\text { resource. }\end{array}$ \\
\hline $\begin{array}{l}\text { S. } \\
\text { Taghizade } \\
\text { h, H. } \\
\text { Bobarshad } \\
\text {, and H. } \\
\text { Elbiaze, } \\
2018\end{array}$ & {$[16]$} & $\begin{array}{l}\text { - Modify of RPL: } \\
\text { - Make high -speed sensor } \\
\text { data stream. } \\
\text { - Increase the lifetime } \\
\text { network. } \\
\text { - Able to balance load and } \\
\text { energy level in the network } \\
\text { system. }\end{array}$ & $\begin{array}{l}\text { - Lifetime } \\
\text { Network } \\
\text { - Load } \\
\text { Balance } \\
\text { - Energy } \\
\text { Efficient }\end{array}$ & $\begin{array}{l}- \text { CAOF } \\
\text { - } \text { CARF } \\
\text { - } \mathrm{RPL} \\
\text { routing } \\
\text { - ETX } \\
\text { metric } \\
\text { - CLRPL }\end{array}$ & $\begin{array}{l}\text { Cooja- } \\
\text { Contiki }\end{array}$ & $\begin{array}{l}\text { - Balance energy } \\
\text { level } \\
\text { - Maximize } \\
\text { lifetime network } \\
\text { - Maximize the } \\
\text { power of the } \\
\text { upstream parent } \\
\text { chain. }\end{array}$ & $\begin{array}{l}\text { - High traffic } \\
\text { load. } \\
\text { - Low } \\
\text { throughput } \\
\text { Network. } \\
\text { - High energy } \\
\text { consumption. }\end{array}$ \\
\hline $\begin{array}{l}\text { A. } \\
\text { Sachan, S. } \\
\text { Nigam, } \\
\text { and A. } \\
\text { Bajpai, } \\
2018\end{array}$ & {$[17]$} & $\begin{array}{l}\text { - Clustering the sensor } \\
\text { network area. } \\
\text { - Used a virtual antenna } \\
\text { technique. } \\
\text { - Design energy resource in } \\
\text { a communication network. }\end{array}$ & $\begin{array}{l}- \text { Energy } \\
\text { consump } \\
\text { tion } \\
\text { - Lifetime } \\
\text { control }\end{array}$ & $\begin{array}{l}\text { - LEACH } \\
\cdot \text { V-MIMO }\end{array}$ & Simulator & $\begin{array}{l}\text { - Boost energy } \\
\text { efficient. } \\
\text { - Increase the } \\
\text { lifetime } \\
\text { network. } \\
\text { - Increase } \\
\text { Performance } \\
\text { Network. }\end{array}$ & $\begin{array}{l}\text { - High energy } \\
\text { consumption. } \\
\text { - Limited } \\
\text { lifetime. } \\
\text { - Overhead of } \\
\text { data } \\
\text { transmission. }\end{array}$ \\
\hline $\begin{array}{l}\text { Y. Cheng, } \\
\text { D. Yang, } \\
\text { and H. } \\
\text { Zhou, } \\
2018\end{array}$ & {$[18]$} & $\begin{array}{l}\text { - Two metric such as miss } \\
\text { packed deadline and ratio } \\
\text { packet loss need to be } \\
\text { considered to remove from } \\
\text { AP's. } \\
\text { - Make sure that station can } \\
\text { be selected by optimal } \\
\text { AP's. }\end{array}$ & $\begin{array}{l}- \text { Load } \\
\text { Balance } \\
\text { - Packet } \\
\text { Loss } \\
\text { - Network } \\
\text { perform } \\
\text { ance }\end{array}$ & $\begin{array}{l}- \text { Det-LB } \\
\cdot \text { RLF } \\
\text { - MMF } \\
\text { - DLBA }\end{array}$ & NS-3 & $\begin{array}{l}\text { - Control packet } \\
\text { loss } \\
\text { performance. } \\
\text { - Improve } \\
\text { network } \\
\text { performance. }\end{array}$ & $\begin{array}{l}\text { - Overloaded } \\
\text { AP's. } \\
\text { - Unbalance } \\
\text { Access points. } \\
\text { - Fixed deadline } \\
\text { - Unbalance } \\
\text { load. }\end{array}$ \\
\hline
\end{tabular}

The overall from the fifteen review papers in Table 1 shows most of $93.33 \%$ of fifteen paper issues due to energy consumption and $73.33 \%$ due to load balancing. Refer to the percentage of the overall paper, can be concluded most of the issues are due to energy consumption and unbalance energy nodes. Where most are affected to maximum the network lifetime and minimize the packet loss performance. Hence, the best performance is come from the minimum energy consumption and reduce traffic during the transmission time. There is a litter bit explanation on both issues in the next subtopic.

\subsection{Energy Consumption}

Energy consumption is the quantity of energy or power used on electronic devices. Where the longer the device is in used, it will increase energy consumption. This issue does not affect to the wired device but its effects on the device that used a battery to support the lifetime. It is because a device that used a battery have limitation support. As mention in section 2, energy consumption is one of the important things that need to be considered to make process perfectly work in Wireless Sensor Network communication. Without enough energy, some data or process cannot be transfer or perform smoothly by a given time.

To make sure lifetime network stay longest, many contributions can be made to achieve the solution for these issues. As a state in Table 1, some of the authors propose to minimize the heavy load [12], [13] balance the energyefficient [15] and used a sleep mode schedule to save the battery power [6].

\subsection{Load Balancing}

In detail explanation, load balancing is the method used to control the flow of the data or packet in the transmission line. The packet needs to be transferred from the sender to the receiver without having any problem. Distance is the main factor in this issue that needs to be measured. Short distance with many data transfer will lead to unbalance traffic.

In balancing the load, it is related to energy consumption, were to control the traffic load in the transmission line, the device needs enough power to transfer data without any delay. Some delay it causes by not enough energy to supply the power to the device. Traffic also can come from the many data have been sending into the transmission line at the same 
time. This issues also has been mention in section 2. All these also can control the packet lost performance as in [18] said in their paper. Both of these issues need to be studied indeeply to resolve all possible problems.

\section{STRENGTH AND LIMITATION OF WSN}

There are several listed of strength and limitation in Wireless Sensor Network can be identified based on previous paper as shown in Table 1. The following subtopics are an explanation of the strengths and limitations of Wireless sensor networks.

\subsection{Strength of WSN}

The strength of the Wireless Sensor Network as identified in Table 1 refer to the terms of functionality, control system, how the management, and the concept idea. The strength of the Wireless Sensor Network are:

\section{- Independent:}

Stand-alone sensor and device become easy to control and manage. Especially when it used in a large of the location area. Where it can operate by itself without using any other support life system.

- Flexible:

The sensor can be placed and remove at any in location range to separate the signal to another nodes sensor.

- Scalability:

Scalable to change the performance from time to time by itself. Especially on manage the traffic load during transmission time. Where the sensor needs to accommodate more nodes while the network is increased.

- Capability:

Able to choose the shortest part direction of the connection to fast delivery and avoid a packet loss.

\subsection{Limitation of WSN}

The limitation listed in Table 1 is referred to as terms of speed, capacity, battery issue, and distraction. The following are the explanation regarding the characteristics are pointed below:

\section{- Capacity:}

The sensor has limited storage where it used to install all the real-time data inside the device. That why nowadays technology combines with the cloud service to manage the data in the Wireless Sensor Network.

\section{- Battery Issue:}

Limited power support makes the life of the sensor depends on the life of the battery. Many functions install in the device makes the sensor used more power to operate.

- Low Speed:
The sensor has limited coverage signal to make some location unable to reach the signal.

\section{- Distraction:}

Due to the fact that many sensors have been built nowadays make the sensor device located everywhere, these make signal will be interrupted by other wireless devices.

\section{CONCLUSION}

In Wireless Sensor Network, energy consumption is one of the important issues need to be stressed it out. From there, many things can be controlled especially to improve the longest network lifetime. Same as balancing of traffic load in the transmission line also need to have thorough research to overcome the packet loss and premature deaths in earlier than other nodes. Many solutions can be performed so that in the future need to be studied in depth to make communication in Wireless Sensor Network work perfectly.

\section{ACKNOWLEDGMENT}

This research was financially supported by the Universiti Putra Malaysia through PUTRA Grant with Project Number GP/2018/9621400.

\section{REFERENCES}

1. J. Zhang, X. Feng, and Z. Liu. A Grid-based clustering algorithm via load analysis for Industrial Internet of Things. IEEE Journal Access, vol.6, no.1, pp.1311713128, Jan 2018. https://doi.org/10.1109/ACCESS.2018.2797885

2. F. Tubiello, L. Poehls, T. Webber, C. Marcon, and F. Vargas. A path energy control technique for energy efficiency in Wireless Sensor Networks. 2018 IEEE 9th Latin American Symposium on Circuits \& Systems (LASCAS) Conference, Puerto Vallarta, 2018 pp.1-4. https://doi.org/10.1109/LASCAS.2018.8399956

3. M. Wollshleager, T. Sauter, and J. Jasperneite. The future of Industrial communication: Automation Networks in the Era of the Internet of Things and Industry 4.0. IEEE Industrial Electronics Journal and Magazine, vol.11, no. 1, pp.17-27, Mar 2017. https://doi.org/10.1109/MIE.2017.2649104

4. B. Malik. Wireless Sensor Network (WSN) and application. Article Microcontroller Lab, Aug 2016.

5. http://microcontrollerslab.com/wireless-sensornetworks-wsn-applications/

6. S. Kaur and R. Goel. A review of data transmission techniques for energy efficiency in Wireless Sensor Networks. 2016 International Conference on Wireless Communications, Signal Processing and Networking (WiSPNET), Chennai, 2016, pp.699-703. https://doi.org/10.1109/WiSPNET.2016.7566223

7. C. Zhan, Y. Zeng, and R. Zhang. Energy-efficient data collection in UAV enabled Wireless Sensor 
Network. IEEE Wireless Communications Letters Journal, vol.7, no.3, pp.328-33, Jun 2018. https://doi.org/10.1109/LWC.2017.2776922

8. I. Khan, and D. Singh. Energy-balance node-selection algorithm for Heterogeneous Wireless Sensor Networks. ETRI Journal, vol.40, no.5, pp.604-612, Oct 2018. https://doi.org/10.4218/etrij.2017-0349

9. J. Ren, Y. Zhang, K. Zhang, A. Liu, J. Chen, and X. Shen. Lifetime and energy hole evolution analysis in data-gathering Wireless Sensor Networks. IEEE Transactions on Industrial Informatics Journal, vol.12, no.2, pp.788-800, Apr 2016. https://doi.org/10.1109/TII.2015.2411231

10. N. Jan, N. Javaid, Q. Javaid, N. Alrajeh, M. Alam, Z. A. Khan, and I. A. Niaz. A balanced energy-consuming and hole-alleviating algorithm for Wireless Sensor Networks. IEEE Access Journal, vol.5, no.1, pp.61346150, Mar 2017. https://doi.org/10.1109/ACCESS.2017.2676004

11. N. Gupta, N. Kumar, and S. Jain. Coverage problem in Wireless Sensor Networks: A survey. 2016 International Conference on Signal Processing, Communication, Power and Embedded System (SCOPES), Paralakhemundi, 2016, pp.1742-1749. https://doi.org/10.1109/SCOPES.2016.7955741

12. M. Faheem, and V. C. Gungor. Energy-efficient and QoS-Aware Routing Protocol for Wireless Sensor Network-based smart grid applications in the context of Industry 4.0. ELSEVIER Journal of Applied Soft Computing, vol.68, no.1, pp.910-922, Jul 2017. https://doi.org/10.1016/j.asoc.2017.07.045

13. X. Li, D. Li, S. Li, S. Wang, and C. Liu. Exploiting industrial big data strategy for load balancing in industrial Wireless Mobile Networks. IEEE Access Journal, vol.6, no. 1, pp.6644-6653, Mar 2018.

14. F. Ren, J. Zhang, T. He, C. Lin, and S. K. Das. EBRP: Energy-Balanced Routing Protocol for data gathering in Wireless Sensor Networks. IEEE Transactions on Parallel and Distributed Systems Journal, vol. 22, no.12, pp.2108-2125, Dec 2011.

15. K. Liu, and C. Wang. Improved the optimal route evaluation method for Wireless Sensor Networks. IEEE Sensors Journal, vol.18, no. 4, pp.1747-1754, Feb 2018.

16. P. Neamatollahi, M. Naghibzadeh, S. Abrishami, and M. H. Yaghmaee. Distributed clustering-task scheduling for Wireless Sensor Networks using dynamic hyper round policy. IEEE Transactions on Mobile Computing Journal, vol.17, no. 2, pp.334-347, Feb 2018.

17. S. Taghizadeh, H. Bobarshad, and H. Elbiaze. CLRPL: Context-Aware and Load balancing RPL for IoT Networks under heavy and highly dynamic Load. IEEE Access Journal, v.6, n.1, pp.23277-23291, Apr 2018. https://doi.org/10.1109/ACCESS.2018.2817128

18. A. Sachan, S. Nigam, and A. Bajpai. An energyefficient Virtual-MIMO communication for clusterbased cooperative Wireless Sensor Network. 2018 9th
International Conference on Computing, Communication and Networking Technologies (ICCCNT), Bangalore, 2018, pp. 1-6. https://doi.org/10.1109/ICCCNT.2018.8493985

19. Y. Cheng, D. Yang, and H. Zhou. Det-LB: A Load Balancing approach in 802.11 Wireless Networks for industrial soft real-time applications. IEEE Access Journal, vol. 6, no. 1, pp. 32054-32063, Feb 2018. https://doi.org/10.1109/ACCESS.2018.2802541 